rectus may be held taut, and if this is so on the right side it is easy to mistake the condition for appendicitis. Tenderness in the hypogastric area is also met with, and the bladder may be full. Any attempt at bimanual palpation of the kidney region may be strongly resented, or even impossible. Examination of the urine reveals that its reaction is acid, and its naked-eye appearance may vary from mere opalescence to thick turbidity from the presence of bacilli, pus, and epithelial debris. The microscope may be necessary, and sometimes we must await the result of cultural investigation.

Another lesion which I must confess I find difficult of diagnosis is pneumococcal peritonitis. This is essentially a children's disease, and it has occurred in infants aged 3 months. It occurs twice as often in girls as in male children, and the common explanation of this is that it is due to an infection from the Fallopian tubes. This I believe to be perfectly true, and I confess that $I$ have rarely diagnosed it correctly except where there has been a vaginal discharge or a definite history of preceding chest trouble. In the latter case the infection is via the blood stream.

Pneumococcal peritonitis presents three definite clinical types-namely:

(1) A severe toxic form with acute onset, diarrhoea, vomiting, and abdominal pain, resembling general peritonitis of appendicular origin.

(2) A subacute type resembling typhoid, with tumid abdomen, regular fever, and constipation.

(3) An almost chronic and insidious form bearing resemblance to tuberculous peritonitis, with abdominal discomfort rather than pain, and slight fever. The bowels may be constipated, but quite often they act regularly.

The physical signs are those of diffuse or local peritonitis, and quite a number of these cases, in whatever way they begin, may settle down to a local intraperitoneal abscess. In the first type the child is desperately ill and the Hippocratic facies may be present, but the surprising thing is that the abdominal rigidity is slight. Examination of the chest does not often reveal evidence of either active or recent disease. Labial herpes is observed in a few cases.

I have already alluded to the presence of vaginal discharge in young girls. It sometimes affords the clue to pneumococcal peritonitis. On the other hand, the organism present may more closely resemble the gonococcus, and true gonorrhoeal peritonitis may be met with in children, but I am afraid I can give you no suggestion as to how often it has been proved to be the cause of peritoneal disease in girls under 12.

Of the rarer varieties of obstruction I have said little. Who can diagnose volvulus, internal hernia, or strangulation through an omental aperture in a child? We may be suspicious. of them, but apart from intussusception the chances in intestinal obstruction in children are in favour of adhesions due to former peritonitis, possibly appendicitis, and those due to a tuberculous gland. I do not think we can hope to get much further. A scar of a previous abdominal operation does help the diagnosis of obstruction, and especially if we know what the scar represents. Remember, also, that recurrent intussusception is not so very rare.

I have explained the need for complete investigation of the cases for the purpose of full and accurate diagnosis so that the necessary operation shall be as brief as possible. This is an ideal we cannot always achieve, and everyone must realize that the limit of his powers may be reached if he separates non-abdominal from true abdominal diseases, and if he is able to differentiate peritonitis from obstruction. Having reached the diagnosis of the "acute abdomen" let us separate the inflammations from the obstructions, and, above all, let us pray that we may avoid the error of opening the abdomen and finding no disease therein.

REFERENCES.

Hamerton : Brjtish Medical Journal, April 25th, 1925.

Acute Abdominal Diseases, second edition, 1924.
CHOLECYSTOGRAPHY. A REPORT OF FIFTY-THREE CASES CONTROLLED BY OPERATION.

BX

\author{
D. P. D. WILKIE, Ch.M., F.R.C.S., \\ PROFESSOR OF SURGERY, EDINBURGII UNIVERSITY; \\ AND
}

O. F. W. ILLINGWORTH, M.B., F.R.C.S.E., CLnICAL TUTOR AND SENIOR CLINICAL ASSISTANT, ROYAL INFIRMARY, EDINBU:GH.

(With Special Plate.)

THe diagnosis of disease of the biliary passages can usually be arrived at with a considerable dewree of accuracy by the ordinary methods of clinical history and examinavion. None the less, the need for accurate methods of physical demonstration such as are now available in urological diagnosis has for long been felt. Whilst with modern technique a radiogram may disclose the presence of gall stones, a greatly thickened gall bladder, or a persistent deformity of the duodenum due to pressure or traction by a diseased gall bladder, the evidence thus obtained is of limited value, as with negative evidence we have no proof that gall stones are not present, nor that we have a healthy and functioning gall bladder.

The demonstration by Rosenthal that in tetrachlor-phenolphthalein we had a substance which was excreted by the liver in the bile, and thus a means of testing liver function, was the basis on which Graham evolved his method of cholecystography. On the grounds that certain of the haloids are opaque to $x$ rays, Graham, in a series of animal experiments, was able to demonstrate that the intravenous injection of calcium tetrabrom-phenol-phthalein and tetraiodo-phenol-phthalein, and in later experiments the sodium salts, was followed in a few hours by the excretion of the salt in the bile and by a visible gall-bladder shadow.

The experiments of Rous and Macmaster had previously proved that the gall bladder had an important function in concentrating the bile. Graham's experiments confirmed this significant observation, for whilst the gall-bladder shadow became more and more intense from the sixth to the eighteenth hour after injection, the ducts were seldom, if ever, visible in the radiograms. Graham showed that, whilst the bromine and iodine salts in large doses were toxic to animals, it was possible to administer a dose sufficient to demonstrate the gall bladder and yet free from dangerous toxic symptoms. A dose of 0.25 gram per kilo of body weight was non-toxic for animals and gave a clear gallbladder shadow. A larger dose caused toxic symptoms. For the human subject a dose of 0.1 gram per kilo of body weight was used. The bromine salt was the less toxic drug, but was somewhat less efficient from the standpoint of radiographic demonstration. Recently Graham has shown that the iodine compound, if carefully prepared, is little, if at all, more toxic than the similar bromine preparation. Milliken and Whitaker report strongly in favour of the iodine salt, which, they maintain, is as safe as, and more efficient than, the bromide. Following Graham's publications, the use of this method in the human subject has now been widely adopted, and evidence is accumulating which goes to show that in cholecystography we have a valuable addition to our methods of physical diagnosis. The largest series of cases reported is that by Carman from the Mayo Clinic, and the evidence from this source is entirely encouraging.

This paper deals with 53 cases, in all of which a cholecystogram was taken prior to an operation on the upper abdomen. The cases included, not only those in which a biliary lesion was suspected, but many in which the dyspeptic symptoms pointed clearly to duodenal or gastric ulcer. We thus obtained a considerable number of controls-an essential in assessing the true value of the method. The method employed in this series of cases was the intravenous injection of sodium tetra-iodo-phenol-phthalein. As results depend largely on the care exercised and the technique employed, we give that used by us in some detail. 
Preparation of the Salt.

For intravenous'injection the salt is dissolved in doubly distilled water, the solution being filtered and sterilized in the autoclave; 5.5 grams of the salt are dissolved in 40 c.cm. of water, and at this dilution the salt readily remains in solution.

\section{Preparation of Patient.}

The usual routine has been as follows: On the day of the injection the patient has his usual breakfast, followed by a light midday meal. The injection has been given in almost all cases between 8 and 10 p.m., and thereafter water only has been allowed until the radiological investigation has been completed. Sodium bicarbonate has been given in all cases, 40 grains being administered every three hours for two days, commencing at midday of the day of injection. As the main reason for limiting the food is to minimizo gall-bladder contractions, and thus to allow lengthy concentration of the bile, it is obvious that no aperient medicine must be given during this period.

Technique of Administration.

Particular care is required during the actual intravenous injection. The fluid is intensely irritating to the subcutaneous tissues, and every effort must be taken to prevent even a single drop escaping outside the vein. To this end it is advisable to introduce the needle into the vein before attaching the syringe. When a good flow of blood has been obtained, the syringe is attached and the fluid very slowly injected. The syringe may then be replaced by a second one containing a few cubic centimetres of saline, which is injected through the needle to prevent any contamination of the tissues during withdrawal. In the earlier cases, following the advice of Graham and Cole, the dose was administered in two equal parts at an interval of half an hour, but in the majority of cases the whole amount has been given at once, and we agree with Milliken and Whitaker that this method presents no disadvantages and is very much more convenient. In about a third of the cases the full dose of 5.5 grams of the salt was administered, dissolved in $40 \mathrm{c.cm}$. of water. In the remainder threc-quarters of this amount was employed. The larger dose ensures a more definite shadow in the healthy caso, and appears to cause little or no more malaise than the smaller dose.

Symptoms Produced by Injection.

Symptoms depending upon the toxicity of the compound have been absent or comparatively mild in the majority of our cases. Of the 45 cases in which the smaller dose (4.125 grams) has been injected, 29 were quite unaffected by the drug, 6 complained of nausea, 9 had nausea and vomiting, only severe in one or two cases. One patient complained of tingling sensation in the lower limbs, coming on immediately after the injection and lasting a short timo only. Of the 23 patients in whom the full 5.5 grams of the drug were einployed, 14 had no symptoms, $6 \mathrm{com}-$ plained of nausea, 2 of nausea and vomiting, and 1 had severe headache lasting for one night. The main indications for the prevention of untoward symptoms would appear to be the adequate restriction of food intake, the administration of alkalis in large quantities, and the slow and careful injection of the diluted solution.

\section{$X$-ray Technique.}

In the majority of the cases, in an effort to simplify the technique as far as possible, a single $x$-ray examination was made, but it is recognized that this procedure has its disadvantages, and that probably the erronoous results obtained in two of our cases would have been avoided had we obtained photographs at different periods. The $x$ rays wero taken at various intervals, from six to twenty-four hours after the injection of the dye, the great majority being taken during the period twelve to fifteen hours, the general impression gained in the earlier observations being that this represented the optimum time.

The usual routine was to take two $x$-ray photographs-one with the patient in the prone position, the tube being directed cbliquely into the right costo-vertebral angle, the other in the lateral direction. The lateral photographs were, however, disappointing, and rarely added to the information gained from the postero-anterior view. In certain suitable cases additional examinations were carried out. In a few the intraperitoneal injection of oxygen was carried out in combination with the cholecystography. In others barium was administered to demonstrate the relationship of gall bladder to pylorus and duodenum, and in yet others a duodenal tube was passed and the effect upon the size of the gall bladder was noted after the injection of magnesium sulphate by the tube.

\section{Interpretation of Radiograms.}

As Graham has pointed out, there are at least three postulates for the production of a well marked gall-bladder shadow, and a consideration of the way in which the opaquo dyo reaches the gall bladder and is amassed in sufficient concentration renders these evident. First, the liver function must be adequate in so far as it concerns the excretion of the opaque salt. It would appear, however, that the margin of safety of liver function is such that considerable impairment of it may exist without materially affecting the result of the test. Secondly, the cystic duct must not be obstructed-for example, by stone, fibrosis, or neoplasm. Thirdly, the concentrating power of the gall bladder must be unaffected. To these three postulates we would add a fourth-namely, that no obstruction must be present in the common bile duct. In our series there are five cases of common duct obstruction-one due to carcinoma of the pancreas and four to stones. In all the cystic duct was patent, yet none of these cases presented a visiblo gall-bladder shadow. The explanation of this is not far to seek: if the obstruction is complete fresh bile cannot enter the ducts from the liver, and if it is only partial, as in many cases of stone in the duct, the concentrating function of the gall bladder is impaired by preceding disease.

In testing such a method the following questions must be answered :

(1) Does a healthy gall bladder invariably give a shadow recognizable as normal?

(2) Does such a "normal" shadow mean a healthy gall bladder?

(3) Do the various pathological lesions of the biliary tract give any characteristic appearances?

Only the cases in which a complete examination of the biliary tract by palpation and inspection was carried out at operation are included in this report. We have divided tho cases into certain groups, which will be doalt with separately.

\section{Grour I.-Normal Gall-Bladder Shadow.} At Operation Biliary Tract Healthy.

In this group there were 23 cases. In all a definite gallbladder shadow of normal outline was visible. The cases included 6 of duodenal ulcer, 2 of gastric ulcer, 4 of chronio appendicitis, 3 of adhesions, and 2 of visceroptosis without organic lesion. The shadow varied with the build of the patient, but was of a density almost comparable to a barium meal. In two cases in which at operation a normal functioning gall bladder was found no shadow had been visible in the radiograms. In both the liver appeared to be normal, and the only explanation available is that food may have been taken between the injection and the radiographio examination. With these two exceptions, the rule was that a healthy gall bladder gave a normal shadow and that a normal shadow indicated a functioning gall bladder (Figs. 1, 2, and 3).

\section{Group II.-No Gall-Bladdfr Shadow.}

At Operation Non-functioning Gall Bladder.

In this group there were 14 cases. In none was any visible shadow cast by the dye, but in 5 cases faint shadowy outlines of gall stones were visible. At operation it was found that in 8 cases the cystic duct was blocked by a calculus impacted either in it or in Hartmann's pouch. In one case the gall bladder was full of stones which prevented the entry of bile although the cystic duct was not obviously obstructed, whilst in 4 cases the gall bladder was shrunken and functionless in association with a stone in the common bile duct. In one case a carcinoma involving the hepatic ducts was assciated with an empty gall bladder.

The lesson to be drawn from this group is that the absence 
of a gall-bladder shadow is not only strong evidence of a gross lesion of the gall bladder but a clear indication for cholecystectomy.

\section{Group III.-Poor Gall-Bladder Shadow.} At Operation Diseased Gall Bladder.

In this group there were 7 cases. In 4 cases gall stones were faintly outlined as negative shadows by the surrounding dye-laden bile (see Fig. 5). In the other 3 the hazy outline and indistinct shadow indicated deficient concentration. In all cases at operation, whilst the gall bladder contained bile, its wall was the seat of gross pathological change and cholecystectomy was performed.

\section{Group IV.-Good Gall-Bladder Shadow + Calculi. At Operation Functioning Gall Bladder.}

In this group there were 3 cases. In one case the gall bladder contained several calculi, but the wall was not thickened and it contained clear bile (sce Fig. 4): In this case an ideal cholecystotomy was performed. In the other two cases the gall-bladder wall, although not functionless, showed naked-eye evidence of pathological change and cholecystectomy was performed.

\section{Group V.-Cases of Obstructive Jaundice.}

The method was employed in 8 cases of obstructive jaundice. Contrary to the experience of Carman, we found no serious untoward effects. In 4 cases there was no reaction, in 4 slight nausea, and in 2 transient vomiting. In no case was any shadow visible in gall bladder or ducts. In 6 of these cases a stone in the common duct was the causative factor, and in 4 of those the gall bladder was shrunken and functionless. In one of the four the whole biliary duct system was distended with " white bile." In two a previous cholecystectomy had been performed. In one case of carcinoma of the head of the pancreas the biliary passages were filled with thick tarry bile which had effectually prevented further biliary excretion. In the last case a carcinoma of the hepatic ducts (already referred to) completely blocked the passage. The lesson to be learned from this group is that in jaundiced patients the method gives little, if any, help in localizing the lesion.

\section{Group VI.-Deformed Gall-Bladder Shadow.} At Operation Local Cholecystitis.

In one case an hour-glass-like shadow in the radiogram was found at operation to be due to a localized hard inflammatory mass in the wall of the gall bladder, the remainder of which appeared healthy (see Fig. 6). A cholecystectomy was performed, and on slitting up the organ a dense fibrous mass, three-quarters of an inch in diameter, was found in an otherwise healthy wall.

\section{Commentary.}

Our experience in this series of cases leads us to believe that in cholecystography we now possess a valuable aid in the diagnosis of gall-bladder disease. When a gall-bladder shadow is visible, if it be of normal density and contour, gross disease may be excluded but mild cholecystitis may be present. Gall stones casting no shadow in the ordinary radiogram may be shown up as negative shadows. Care must, however, be exercised not to mistake gas in the overlying colon for a gall stone. Given correct technique the absence of a gall-bladder shadow indicates one of three conditions :

(1) Obstruction of the cystio duct by a stone within it or in Hartmann's pouch, or by fibrosis, catarrh, or neoplasm.

(2) A gall bladder so filled by stones that dye-laden bile cannot enter.

(3) A gall bladder so diseased or shrunken as to be functionless.

In cases where a preliminary radiogram has shown doubtful shadows suggestive of gall stones, the accurate localization of the gall bladder gives confirmatory evidence, the method being thus comparable to pyelography in cases of renal calculus. In such a case, under the eare of Mr. Henry Wade, the preliminary $x$-ray photograph showed what appeared to be a renal calculus as well as gall stones. Tho sites of the calculi were accurately defined by pyelography and cholecystography, and both conditions successfully dealt with at one operation. When a palpable lump is present in the right hypochondriac region it is possible by cholecystography to determine whether it is a new growth in the liver or a distended gall bladder. In a case which was lately under our care the patient had recently been operated on for an endothelioma of the thigh. He developed a painless, rounded, and mobile swelling under the right costal margin associated with an intermittent rise of temperature. A cholecystogram showed that the gall bladder was functioning normally and was situated below the margin of the swelling, thus confirming the diagnosis of secondary growth in the liver.

In order to simplify the test the dye may be given by the mouth, either in capsules or in pill form. Carman has practised this method of administration extensively in the Mayo Clinic and recommends it for general use. We have used the oral method in but a few cases. The results were so inferior to those obtained by the intravenous administration of the dye and the latter so much more accurate and certain that we now practise it exclusively.

The outstanding feature of cholecystography is that it is a means, not only of demonstrating anatomico-pathological changes in the gall bladder, but also of testing the functional capacity of the organ. Whilst modifications and improvements of the method will doubtless follow further experience, we believe that it will have a permanent place as a method of physical diagnosis.

In conclusion we desire to express our thanks to $\mathrm{Mr}$. Henry Wade for permission to include three cases which were under his care in this series, and to Mr. Leslie Stewart for his help in the investigation of these cases.

\section{REFERENCES.}

1 Graham and Cole: Journ. Amer. Med. Assoc., 1924, lxxxii, 63. 2 Graham, Cole, and Copher: Ibid,, 1924, Ixxxii, 1777. 3 Graham, Cole and Copher: Annals of Surgery. September, 1924, 473. ${ }_{3}^{4}$ Graham, Cole, and Copher: Journ. Amer. Med. As8oc., 1925, Jxxxiv, 14. 5 Whitaker and Milliken S Surg., Gynecol. and Obstet., January, 1925. 'Carman: 1925, ii, 54.

\section{EMBRYOMA OF THE TESTIS :}

Sudden Death from Thrombosis of Pulmonary Veins.

$$
\text { (With Special Plate.) }
$$

BY

A. E. WEBB-JOHNSON, C.B.E., D.S.O., M.B., F.R.C.S., F.A.C.S.,

SURGEON TO THE MIDDLESEX HOSPITAL.

Ir is not at all uncommon for a patient with a tumour of the testis to seek advice in the first instance on account of secondary deposits, either in the loin or even in the posterior triangle of the neck when metastases have travelled along the thoracic duct; but the extreme case of death occurring without any tumour having been noticed must be almost unique.

An officer, aged 31, was admitted to No. 14 General Hospital, Boulogne Base, on November 15th, 1915, as a "walking case," Boulogne Base, on down from the front with a diagnosis of "lumbago." He was admitted in the late evening, and went to "lumbago." He was admitted in the late evening, and we complained of pain in the right lumbar region, which bed. He complained of pain in the right lumbar region, which he said was proving distinctly trymg under the conditions of lite think the pain would have worried him. He stated that he only had it on povement and that he was quite comfortable when in had it on duty until November 5th. For two or three days after on duty until November 5 th. For two or three

reporting sick he had a slight rise of temperature.

The morning after admission to the base hospital he was up and about, attending to his toilet and visiting some of his friends, who were in neighbouring rooms. As he was talking to a brother offer he she medical officer saw him immediately, and within a fow me several of us in attendance. In spite a fow soon obvious of overy effort to resud in a few panutes His appearance was that he would be dead in a few pilnutes. His appearance was that of a man receiving no oxygen into his circulation, although there was some shallow respiration. The pulse rapidly failed, and within about ten minutes of the appearance of symptoms he was dead. A post-mortem exammation was made, and smce his symptoms were suggestive of pulmonary thrombosis or embolism the thorax was examined first. The lungs and heart were removed ho ther, asterior mediastinum. together, along with the conts The pulmonary veins were opcned, almost gelatinous, mass, about 